Research Article

\title{
The Existence and Effect of Dark Energy Redshift on Cosmological Age
}

\author{
Riccardo C. Storti iD \\ Delta Group Engineering, Melbourne, Australia \\ Correspondence should be addressed to Riccardo C. Storti; zordan@ozemail.com.au
}

Received 17 February 2020; Accepted 15 June 2020; Published 11 August 2020

Academic Editor: Roberto Turolla

Copyright (C) 2020 Riccardo C. Storti. This is an open access article distributed under the Creative Commons Attribution License, which permits unrestricted use, distribution, and reproduction in any medium, provided the original work is properly cited.

A derivation of Cosmological Age explicitly constrained by Cosmic Microwave Background Radiation (CMBR) is presented, demonstrating that the correct value of Cosmological Age is equal to the Hubble Age. It is shown that utilizing " $z=0$ " for Cosmological Redshift in the Present Epoch introduces a fundamental flaw into Cosmological Age calculations. However, this flaw is captured and corrected by the Polarizable-Vacuum (PV) Model of Gravity developed by Puthoff, suggesting that the Dark Energy Field exists as a massive photonic field. Consequently, it is demonstrated that for a Dark Energy Driven description of Accelerated Cosmological Expansion, Cosmological Redshift takes a negative value in the Present Epoch.

\section{Dilemma}

1.1. Introduction. The Standard Model of Cosmology (SMoC) utilizes Cosmic Microwave Background Radiation (CMBR) data to determine the age of the Universe, defined by [1] as 13.797 (Gyr) \pm 23 (Myr). However, notably in contrast, the age of star HD-140283 is defined in [2] as 14.46 (Gyr) \pm 800 (Myr). Although no emphatic contradiction exists between these two observations, due to the associated experimental tolerances, it provokes scrutiny as the HD-140283 lower observational limit is 13.66 (Gyr). Since the lower CMBR limit is 13.774 (Gyr), an arguably narrow 114 (Myr) congruence window exists between CMBR and HD-140283 measurements. Cognizant of the exponential rate of technological growth, one may well expect that the tolerance commonality between these two experimental sources might instantly vanish. For such an event to occur, the SMoC may require significant revision.

It seems improbable that the physical measurements gathered by the instrumentation utilized in the Planck Satellite [3] are flawed; the Science and Engineering underlying the technology is well-understood, and significant instrumentation defects would have become rapidly evident and subsequently corrected prior to deployment. It is equally improbable that the software associated with the observation and data collection processes might lead to incorrect results as the computational output is broadly peer-reviewed prior to publication. Thus, by elimination, the remaining candidate for scrutiny is the theoretical basis from which the final age determination is executed according to the SMoC relationship

$$
-\frac{1}{H_{0}} \int_{\infty}^{Z} \frac{1}{(1+z) \sqrt{\left(1-\Omega_{\Lambda}\right)(1+z)^{3}+\Omega_{\Lambda}}} \mathrm{d} z=13.797 \mathrm{Gyr},
$$

where [1] $H_{0}=$ Hubble Constant $=67.4(\mathrm{~km} / \mathrm{s} / \mathrm{Mpc}) \pm 0.5$ $(\mathrm{km} / \mathrm{s} / \mathrm{Mpc})$ and $\Omega_{\Lambda}=$ Dark Energy Density Parameter $=$ $0.685 \pm 0.007$.

If the Cosmological Redshift for the Present Epoch is assigned the value " $z=0$," (1) simplifies to

$$
\frac{2}{3 H_{0} \cdot \sqrt{\Omega_{\Lambda}}} \cdot \ln \left(\frac{1+\sqrt{\Omega_{\Lambda}}}{\sqrt{1-\Omega_{\Lambda}}}\right)=13.797 \mathrm{Gyr}
$$

It is important to note that " $z=0$ " for the Present Epoch is an assigned value, not an assumed value. An assigned value is a man-made contrivance, whilst an assumed value can have natural origins. The assigned value for the Present Epoch seems reasonable, benign, and convenient, but what 
are the implications associated with this? To address this question and validate (2), we require an alternative representation of Cosmological Redshift. Fortunately, Puthoff [4] developed an alternative representation of General Relativity (GR) termed The Polarizable-Vacuum (PV) Model of Gravity. Since then, Depp et al. $[5,6]$ and Desiato [7] have shown the PV Model of Gravity to be isomorphic to GR by satisfying the Schwarzschild and Reissner-Nordstrom solutions.

1.2. The PV Model. The PV Model of Gravity is an optical derivative, utilizing a Refractive Index value " $K_{\mathrm{PV}}$." For an observer at the bottom of a gravity well, gazing upwards and backwards in time (e.g., at the surface of the Earth), the relationship between Cosmological Redshift " $\mathrm{z}$ " and " $K_{\mathrm{PV}}$ " utilizing Puthoff relationships [4] is given by

$$
1+z=\frac{1}{\sqrt{K_{\mathrm{PV}}}} .
$$

Hence, (1) expressed in PV form is given by

$$
\frac{1}{2 H_{0}} \int_{0}^{1} \frac{1}{K_{\mathrm{PV}} \sqrt{\left(1 / \sqrt{K_{\mathrm{PV}}^{3}}\right)\left(1-\Omega_{\Lambda}\right)+\Omega_{\Lambda}}} d K_{\mathrm{PV}}=13.797 \mathrm{Gyr} .
$$

$(1)=(4)$; therefore, the PV Model of Gravity aligns precisely to the SMoC (as expected). However, (4) reveals an important fact regarding the assigned value of Cosmological Redshift " $z=0$," utilized to formulate (2). That is, the Refractive Index value " $K_{\mathrm{PV}}=1$ " can only occur when the observer possesses the power of infinite gaze in our Universe or resides at infinity outside our Universe (e.g., in a Multiverse Cosmological Model). This means that the assigned value of Cosmological Redshift " $z=0$ " represents a nonphysical situation, thereby denoting an invalid procedural step. Utilizing " $z=0$ " requires The Observable Universe to be infinitely large, yet CMBR observations are seeking to measure a finite size. Hence, (2) is in conflict with CMBR experimental objectives, demonstrated by the Gravitational Refractive Index as implicitly asserted by Desiato [7], such that the value " $K_{\mathrm{PV}}=1$ " occurs as " $r \longrightarrow \infty$ " according to

$$
K_{\mathrm{PV}}=\frac{1}{\left(1-\left(2 G \cdot M / r \cdot c^{2}\right)\right)}=\frac{r \cdot c^{2}}{r \cdot c^{2}-2 G \cdot M} .
$$

Note: equation (5) represents the Metric Coefficient of the Schwarzschild Solution of Einstein's equations of GR.

\section{Resolution}

Equation (5) invokes the question if " $r \longrightarrow \infty$ " should not be applied, what should be? To address this question, we shall conduct the following thought experiment: consider an Observable Universe of Hubble Radius " $R_{H}$." For an observer to circumnavigate this Universe, he/she must travel a distance of " $2 \pi R_{H}=2 \pi c / H_{0}$ "; hence, from the perspective of the observer, (5) is required to metabolize the condition " $r=2 \pi c / H_{0}$." Moreover, it follows that if the Universe is of Hubble Radius, then " $M$ " relates to Hubble Volume; hence, " $M$ " is equal to the Dark Energy Density Parameter multiplied by the Hubble Volume multiplied by the Critical Density, such that a Dark Energy Refractive Index " $K_{\Lambda}$ " may be formulated according to

$$
K_{\Lambda}=\frac{\left(2 \pi c / H_{0}\right) c^{2}}{\left(2 \pi c / H_{0}\right) c^{2}-2 G\left(\Omega_{\Lambda}\left[(4 / 3) \pi\left(c / H_{0}\right)^{3}\right]\left(3 H_{0}^{2} / 8 \pi G\right)\right)}=\frac{2 \pi}{2 \pi-\Omega_{\Lambda}} .
$$

Substituting (6) into (3) yields the Dark Energy Redshift " $z_{\Lambda}$ " as follows:

$$
z_{\Lambda}=\sqrt{1-\frac{\Omega_{\Lambda}}{2 \pi}}-1
$$

Combining (6) with PV Time Dilation of the form " $\Delta t_{0}=\Delta t \cdot \sqrt{ } K_{\mathrm{PV}}$ " as defined by Puthoff [4] yields a revised expression for Cosmological Age " $t_{0}$ " as follows:

$$
t_{0}=\frac{2}{3 H_{0} \sqrt{\Omega_{\Lambda}}} \ln \left[\frac{1+\sqrt{\Omega_{\Lambda}}}{\sqrt{1-\Omega_{\Lambda}}}\right] \cdot \sqrt{\frac{2 \pi}{2 \pi-\Omega_{\Lambda}}} .
$$

\section{Results}

3.1. Theoretical Solution. Equation (6) was formulated for a Theoretical Universe of Hubble Radius; hence, for the Hubble Time configuration $t_{0}=1 / H_{0}$, we obtain the following outputs: (i) $\Omega_{\Lambda}=0.677345709533812$

(ii) $\Omega_{m}=0.322654290466188$

(iii) $K_{\Lambda}=1.12082859270862$

(iv) $z_{\Lambda}=-0.055438153350662$

The following are the key points:

(i) Our Theoretical Universe is a useful representation of physical reality

(ii) Our $\Omega_{\Lambda}$ lies less than “0.1 (\%)" outside SMoC limits (a) The SMoC range of $\Omega_{\Lambda}$ is “ $0.685 \pm 0.007$ ” [1]

(iii) The proximity of our $\Omega_{\Lambda}$ to SMoC results denotes initial evidence that the Hubble Age (8) is the correct Cosmological Age; not (2)

(iv) $K_{\Lambda}$ is greater than unity, denoting Accelerated Cosmological Expansion 
(v) $z_{\Lambda}$ is negative, denoting Accelerated Cosmological Expansion

(vi) Calculations utilizing an assigned Cosmological Redshift of $z=0$ may require revision

3.2. Constrained Theoretical Solution. For the Hubble Time configuration $t_{0}=1 / H_{0}$ constrained to CMBR Temperature in accordance with the relationship formulated by Storti [8-11],

$$
T_{0}=K_{W} S t_{T} \cdot \ln \left(\frac{H_{\alpha}}{H_{0}}\right) H_{0}^{5 \mu^{2}},
$$

where $H_{\alpha}=$ Big-Bang Hubble Constant $=8.46087689814736$ $\times 10^{61}(\mathrm{~km} / \mathrm{s} / \mathrm{Mpc})$, the derivation of $H_{\alpha}$ can be found on $\mathrm{pg}$. 12, Equation (24); [10], $S t_{T}^{9}=$ Physical Constant $=$ $6.35538654769188 \times 10^{95}\left(\mathrm{~s}^{5} / \mathrm{m}^{9}\right)$, the derivation of $S t_{T}$ can be found on pg. 14, Equation (42); [10], $K_{W}=$ Wien Displacement Constant $=2.897771955(\mathrm{~mm} \cdot \mathrm{K}), \mu=$ Mathematical Constant $=1 / 3, T_{0}=2.7255(\mathrm{~K}) \pm \Delta T_{0}[1]$, and $\Delta T_{0}=$ $0.0006(\mathrm{~K})$.

Note: $H_{\alpha}$ and $S t_{T}$ are forwardly derived from first principles; they are not reverse-engineered, ad hoc, or tailored constants; refer to [10] for the derivation.

The calculated permissible range values for " $\Omega_{\Lambda}, \Omega_{m}, K_{\Lambda}$, $z_{\Lambda}, H_{0}$ and $t_{0}$ " are displayed in Table 1 .

The following are the key points:

(i) Our CMBR Temperature-constrained Theoretical Universe remains a useful representation of physical reality

(ii) $\Omega_{\Lambda}, \Omega_{m}, K_{\Lambda}$, and $z_{\Lambda}$ remain constant across the CMBR observational range

(iii) $\Omega_{\Lambda}$ lies less than "0.1 (\%)" outside SMoC limits

(iv) $H_{0}=67.1181(\mathrm{~km} / \mathrm{s} / \mathrm{Mpc}) \pm 0.0269(\mathrm{~km} / \mathrm{s} / \mathrm{Mpc})$

(a) $H_{0}$ lies within $\mathrm{SMoC}$ observational limits

(b) $H_{0}$ resolution is improved by one order-ofmagnitude

(c) Our value of $H_{0}$ validates the SMoC-expected (unproven) dependency between the Hubble constant and CMBR Temperature

(d) Our value of $H_{0}$ denotes supporting evidence for the Hubble Age (8) being the correct Cosmological Age; not (2)

(v) $t_{0}=14.5685(\mathrm{Gyr}) \pm 5.849(\mathrm{Myr})$

(a) Our value denotes unprecedented resolution of Cosmological Age

(b) We show synergy with HD-140283:14.46 (Gyr) \pm 114.39 (Myr)

(c) Our value reduces the potential for a "Crisis in Cosmology"

3.3. Heuristic Solution. For the Hubble Time configuration $t_{0}=1 / H_{0}$ utilizing key SMoC data ranges [1], we calculate the outputs as displayed in Table 2.
The following are the key points:

(i) The SMoC determination of Cosmological Age [1] does not concur with CMBR Temperature observations (i.e., $t_{0}$ is in conflict with $T_{0}$ )

(a) The use of (9) provides supporting evidence for the Hubble Age (8) being the correct Cosmological Age, not (2)

(ii) $\mathrm{SMoC}$ Hubble Constant observations are consistent with CMBR Temperature observations, validating (9)

(iii) SMoC Dark Energy Density values are consistent with CMBR Temperature observations, validating (9)

(iv) Our CMBR Temperature-constrained Theoretical Universe remains a useful representation of physical reality

3.4. Constrained Heuristic Solution. For the Hubble time configuration $t_{0}=1 / H_{0}$ constrained by SMoC $\Omega_{\Lambda}$ and $T_{0}$ values [1], we calculate the outputs as displayed in Table 3.

The following are the key points:

(i) Many SMoC combinations of $\Omega_{\Lambda}$ and $H_{0}$ exist to satisfy observational CMBR Temperature requirements, validating (9)

(ii) The SMoC " $\Omega_{\Lambda}+\Delta \Omega_{\Lambda}$ " limit violates the SMoC " $H_{0}+\Delta H_{0}$ " limit

(iii) Our dark energy plus CMBR Temperature-constrained Theoretical Universe is a useful representation of physical reality

\section{Discussion}

The preceding analysis clearly demonstrates the correlation between physical reality and our Theoretical Universe of Hubble Radius, offering a persuasive argument supporting the contention that the Hubble Age is the true Cosmological Age, not the present value as calculated by the SMoC [1]. So, why is the SMoC determination of Cosmological Age inaccurate? Primarily, it is because the method of calculation does not include a direct and clear relationship to CMBR Temperature. Cosmological Age, Hubble Constant, Dark Energy and CMBR Temperature must be related, yet The SMoC solution only embraces three of the four critical elements for physical meaningfulness. This is the first clue as to why the SMoC determination of Cosmological Age is inaccurate. The second clue is in the fact that the $\mathrm{SMoC}$ value is 663 (Myr) less than the value for HD-140283. Although observational tolerances ensure that the results are not in conflict in this case, it is prudent for us to emphasize the meaning of error bars. Error bars provide no guarantee that the central value is not incorrect to begin with, only that the central value lies between certain limits. The assertion of experimental consistency between two data sets based solely upon tolerance overlap is not a robust defence. If either 
TABLe 1: $H_{0}(\mathrm{~km} / \mathrm{s} / \mathrm{Mpc})$ and $t_{0}(\mathrm{Gyr})$.

\begin{tabular}{lccc}
\hline & $T_{0}-\Delta T_{0}$ & $T_{0}$ & $T_{0}+\Delta T_{0}$ \\
\hline$\Omega_{\Lambda}$ & 0.677345709533813 & 0.677345709533812 & 0.677345709533812 \\
$\Omega_{m}$ & 0.322654290466187 & 0.322654290466188 & 0.322654290466188 \\
$K_{\Lambda}$ & 1.12082859270862 & 1.12082859270862 & 1.12082859270862 \\
$z_{\Lambda}$ & -0.055438153350662 & -0.055438153350662 & -0.055438153350662 \\
$H_{0}$ & 67.0912006738519 & 67.1181447977434 & 67.1450938090621 \\
$t_{0}$ & 14.5743867417478 & 14.5685359530647 & 14.5626888000112 \\
\hline
\end{tabular}

TABle 2: $T_{0}(K)$

\begin{tabular}{lccc}
\hline & $T_{0}$ & Configuration & Methodology \\
\hline$t_{0}-\Delta t_{0}$ & 2.81061377079329 & & Direct injection into $(9): T_{0}\left(1 / t_{0}\right)$ \\
$t_{0}$ & 2.80804368741903 & $t_{0}=13.797(\mathrm{Gyr}) \pm \Delta t_{0}, \Delta t_{0}=23(\mathrm{Myr})$ & \\
$t_{0}+\Delta t_{0}$ & 2.80548022874534 & & Direct injection into $(9): T_{0}\left(H_{0}\right)$ \\
\hline$H_{0}-\Delta H_{0}$ & 2.72063915377994 & & \\
$H_{0}$ & 2.73176992769907 & $H_{0}=67.4(\mathrm{~km} / \mathrm{s} / \mathrm{Mpc}) \pm \Delta H_{0}, \Delta H_{0}=0.5(\mathrm{~km} / \mathrm{s} / \mathrm{Mpc})$ & \\
$H_{0}+\Delta H_{0}$ & 2.74286346018614 & & \\
\hline$\Omega_{\Lambda}-\Delta \Omega_{\Lambda}$ & 2.73082285014193 & & \\
$\Omega_{\Lambda}$ & 2.7206171322242 & $H_{0}=67.4(\mathrm{~km} / \mathrm{s} / \mathrm{Mpc}), \Omega_{\Lambda}=0.685 \pm \Delta \Omega_{\Lambda}, \Delta \Omega_{\Lambda}=0.007$ & Injection into $(8)$, and then into $(9): T_{0}\left(1 / t_{0}\right)$ \\
$\Omega_{\Lambda}+\Delta \Omega_{\Lambda}$ & 2.71027372079337 & & \\
\hline
\end{tabular}

central value evolves from a fundamentally incorrect approach, the tolerance overlap is valueless.

Our significant deviation from SMoC doctrine warrants explanation, satisfying the question as to how this oversight occurred. Herein, we have identified the cause $(z=0)$, proposed a resolution $z_{\Lambda}$, and quantified the outcome $\Omega_{\Lambda}$. The next logical question to investigate is why an assigned value of $z=0$ is inappropriate to apply, that is, why is (2) inaccurate? Considering the synergy between our
Theoretical Universe and physical reality, we propose that (2) is incomplete because it does not adequately consider the time dilation effects of a Massive Photonic Field.

We propose that the Dark Energy Field is a massive photonic field

Evidence for this claim arose in 2008, where Storti [8] accurately calculated CMBR Temperature utilizing (9) and predicted the value of the Hubble Constant in advance of experimental measurement:

$$
H_{0}=\frac{m_{\gamma \gamma}}{h} \cdot \ln \left(\frac{\sqrt{(3 \pi)^{7 \mu} \cdot 32^{\mu^{2}}}}{256} \cdot \ln \left(\frac{(3 \pi)^{\mu}}{4} \cdot\left(\frac{m_{h}}{M_{G}}\right)^{\mu^{2}}\left(\frac{R_{0}}{\lambda_{h}}\right)^{7 \mu^{2}}\right)^{7 \mu} \cdot\left(\frac{m_{h}}{M_{G}}\right)^{5 \mu^{2}}\left(\frac{R_{0}}{\lambda_{h}}\right)^{26 \mu^{2}}\right)^{5}
$$

where $m_{\gamma \gamma}=$ Photon Mass-Energy $=3.19515507344683 \times$ $10^{-45}(\mathrm{eV})$ [12], $h=$ Planck's Constant $=6.62607015 \times 10^{-34}$ $(\mathrm{J} / \mathrm{Hz}), \quad m_{h}=$ Planck Mass $=5.45551186133462 \times 10^{-8}(\mathrm{~kg})$, $\lambda_{h}=$ Planck Length $=4.05135054323488 \times 10^{-35}(\mathrm{~m}), M_{G}=$ Galactic Mass (2008: predicted $)=6.3142 \times 10^{11}\left(M_{S}\right)$, and $\mu=$ Mathematical Constant $=1 / 3$.

The yielded predictions are displayed in Table 4.

The following are the key points:

(i) Equations (9) and (10) have been experimentally verified

(ii) Equation (10) utilizes a derived value of nonzero Photonic Mass-Energy

(iii) The existence of a Massive Photonic Dark Energy Field is implied

(iv) The Massive Photonic Dark Energy Field is responsible for Accelerated Cosmological Expansion (v) The effect of Accelerated Cosmological Expansion is to "pull" time dilation in the "opposite direction," acting to "stretch time"

(vi) Cosmological Inflation and Accelerated Cosmological Expansion are derived naturally and without "fine tuning" or "retro-fitting" in [8-11].

(vii) The true value of Cosmological Age equals the Hubble Age

Upon consideration of all results presented herein, we predict that future experimental observations will tend to yield values of Dark Energy Density Parameter increasingly approaching the present SMoC lower limit " $\Omega_{\Lambda}-\Delta \Omega_{\Lambda}$ ". Moreover, we propose that the difference between our theoretical $\Omega_{\Lambda}$ value and the present SMoC lower limit may represent a possible candidate for spatial curvature $\Omega_{K}$, as follows:

(i) The present $\mathrm{SMoC} \Omega_{\Lambda}$ lower limit $=$ $0.685-0.007=0.678$ 


\begin{tabular}{|c|c|c|c|}
\hline \multicolumn{4}{|c|}{$\Omega_{\Lambda}-\Delta \Omega_{\Lambda}$} \\
\hline & $T_{0}-\Delta T_{0}$ & $T_{0}$ & $T_{0}+\Delta T_{0}$ \\
\hline$H_{0}$ & 67.1336409514318 & 67.160602119527 & 67.1875681781412 \\
\hline$t_{0}$ & 14.5651731640227 & 14.5593260740679 & 14.5534826174442 \\
\hline \multicolumn{4}{|c|}{$\Omega_{\Lambda}$} \\
\hline & $T_{0}-\Delta T_{0}$ & $T_{0}$ & $T_{0}+\Delta T_{0}$ \\
\hline $\mathrm{H}_{0}$ & 67.593627468596 & 67.6207733693176 & 67.6479241940671 \\
\hline$t_{0}$ & 14.4660546002391 & 14.4602473007592 & 14.4544436098851 \\
\hline \multicolumn{4}{|c|}{$\Omega_{\Lambda}+\Delta \Omega_{\Lambda}$} \\
\hline & $T_{0}-\Delta T_{0}$ & $T_{0}$ & $T_{0}+\Delta T_{0}$ \\
\hline $\mathrm{H}_{0}$ & 68.0648179486208 & 68.09215308153 & 68.1194931727921 \\
\hline$t_{0}$ & 14.365910834097 & 14.3601437366527 & 14.3543802228329 \\
\hline
\end{tabular}

TABLE 4: Experimental validation of predictions.

\begin{tabular}{lcc}
\hline PDG (2008) [13] & $\begin{array}{c}\text { Storti }(2008)[8,9] \\
\text { Prediction }\end{array}$ & $\begin{array}{c}\text { PDG (2019) [1] } \\
\text { Measurement }\end{array}$ \\
\hline$H_{0}=73(\mathrm{~km} / \mathrm{s} / \mathrm{Mpc})$ & $H_{0}=67.0843(\mathrm{~km} / \mathrm{s} / \mathrm{Mpc})$ & $\begin{array}{c}H_{0}=67.4 \\
(\mathrm{~km} / \mathrm{s} / \mathrm{Mpc})\end{array}$ \\
$R_{0}=8.0(\mathrm{kpc})$ & $R_{0}=8.1072(\mathrm{kpc})$ & $R_{0}=8.178(\mathrm{kpc})$ \\
$T_{0}=2.725(\mathrm{~K})$ & $T_{0}=2.7248(\mathrm{~K})$ & $T_{0}=2.7255(\mathrm{~K})$ \\
\hline
\end{tabular}

(ii) The present SMoC value of spatial curvature $\Omega_{K}=0.0007$ [1]

(iii) Our value for $\Omega_{\Lambda}$ in the theoretical solution $=$ 0.677345709533812

(iv) Our value for $\Omega_{K}=0.678-0.677345709533812=$ 0.000654290466188

The following are the key points:

(i) Our value for $\Omega_{K}$ is quasi-identical to the $\mathrm{SMoC}$ value

\section{Conclusions}

The Polarizable-Vacuum Model of Gravity developed by Puthoff [4], the isomorphic work presented by Dep et al. [5, 6], and the form of the Gravitational Refractive Index implicitly asserted by Desiato [7] have enabled us to refine the estimate for Cosmological Age in the Standard Model of Cosmology.

The following are the key points:

(i) The true value of Cosmological Age equals the Hubble Age

(ii) Calculations utilizing an assigned Cosmological Redshift of " $z=0$ " may require revision

(iii) The value of the Cosmological Refractive Index is greater than unity for the Present Epoch, denoting Accelerated Cosmological Expansion

(iv) The value of Cosmological Redshift is negative for the Present Epoch, denoting Accelerated Cosmological Expansion

(v) The SMoC determination of Cosmological Age does not concur with CMBR Temperature observations (i.e., $t_{0}$ is in conflict with $T_{0}$ ) (vi) SMoC Hubble Constant observations are consistent with CMBR Temperature observations

(vii) SMoC Dark Energy Density values are consistent with CMBR Temperature observations

(viii) The SMoC " $\Omega_{\Lambda}+\Delta \Omega_{\Lambda}$ " limit violates the SMoC " $H_{0}+\Delta H_{0}$ " limit

(ix) The existence of a Massive Photonic Dark Energy Field is implied

(x) The Massive Photonic Dark Energy Field is responsible for Accelerated Cosmological Expansion

(xi) The effect of Accelerated Cosmological Expansion is to "pull" time dilation in the "opposite direction," acting to "stretch time"

(xii) $H_{0}=67.1181(\mathrm{~km} / \mathrm{s} / \mathrm{Mpc}) \pm 0.0269(\mathrm{~km} / \mathrm{s} / \mathrm{Mpc})$

(xiii) $t_{0}=14.5685(\mathrm{Gyr}) \pm 5.849$ (Myr)

\section{Data Availability}

Full calculations are supplied via a solution algorithm; all data sources are referenced and available within the article.

\section{Conflicts of Interest}

The authors declare that they have no conflicts of interest.

\section{Supplementary Materials}

The supplementary materials contain the calculation of the Existence and Effect of Dark Energy Redshift on the Cosmological Age and Minimised Solution Algorithm (cosmology). (Supplementary Materials)

\section{References}

[1] PDG, “Astrophysical constants and parameters," 2019, http://pdg. lbl.gov/2019/reviews/rpp2019-rev-astrophysical-constants.pdf.

[2] H. E. Bond, E. P. Nelan, D. A. VandenBerg, G. H. Schaefer, and D. Harmer, "HD 140283: a star in the solar neighborhood that formed shortly after the Big bang," 2013, https://arxiv. org/abs/1302.3180.

[3] N. Aghanim, Y. Akrami, and M. Ashdown, "Planck 2018 results. VI. Cosmological parameters," 2018, https://arxiv.org/ abs/1807.06209.

[4] H. E. Puthoff, "Polarizable-Vacuum (PV) representation of general relativity," 1999, https://arxiv.org/ftp/gr-qc/papers/ 9909/9909037.pdf.

[5] J. G. Depp, T. J. Desiato, and R. C. Storti, "Polarizable vacuum and the Schwarzschild solution," 2005, https:// www.researchgate.net/publication/339998850_Polarizable_ Vacuum_and_the_Schwarzchild_Solution.

[6] J. G. Depp, T. J. Desiato, and R. C. Storti, "Polarizable vacuum and the reissner-nordstrom solution," 2005, https:// www.researchgate.net/publication/339999018_Polarizable_ Vacuum_and_the_Reissner-Nordstrom_Solution.

[7] T. J. Desiato, "General relativity and the polarizable vacuum," 2006, https://www.researchgate.net/publication/304460849_ General_Relativity_and_the_Polarizable_Vacuum.

[8] R. C. Storti, "The natural philosophy of the cosmos (A)," in Proceedings of the 18th National Congress, Australian Institute 
of Physics, Adelaide, Australia, 2008, https://www.researchgate. net/publication/272301444_The_Natural_Philosophy_of_The_ Cosmos_A.

[9] R. C. Storti, "The natural philosophy of the cosmos (B)," in Proceedings of SPIE-The International Society for Optical Engineering, vol. 7421, San Diego, California, USA, September 2009, https://www.researchgate.net/publication/253296652 The_Natural_Philosophy_of_The_Cosmos_BThe Nature of Light: What are Photons? III, $74210 \mathrm{H}$.

[10] R. C. Storti, "The Natural Philosophy of the Cosmos (C)," in Quinta Essentia: A Practical Guide to Space-Time Engineering: Part 4, pp. 175-193, 2008, 2nd edition, https://www.researchgate. net/publication/272498371_The_Natural_Philosophy_of_the_Co smos_C7.

[11] R. C. Storti, "Quinta essentia: part 5: a practical guide a practical guide to space-time engineering (solution algorithm)," 2020, https://www.researchgate.net/publication/ 335173066_Quinta_Essentia_Part_5_A_Practical_Guide_to_ Space-Time_Engineering_Solution_Algorithm.

[12] T. J. Desiato and R. C. Storti, "Derivation of the Photon and graviton mass-energies and radii," The Nature of Light: What Is a Photon? in Proceedings of the SPIE 5866, vol. 5866, p. 214, August 2005, https://www.researchgate.net/publication/ 252465171_Derivation_of_the_Photon_and_Graviton_MassEnergies_and_Radii.

[13] Particle Data Group, http://pdg.lbl.gov/2008/reviews/rpp2008rev-astrophysical-constants.pdf, 2008. 